Journal of the

Optical Society

of America

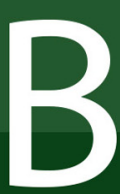

OPTICAL PHYSICS

\title{
Generalized 4 x 4 matrix formalism for light propagation in anisotropic stratified media: study of surface phonon polaritons in polar dielectric heterostructures: erratum
}

\author{
Nikolai Christian Passler* (i) and Alexander PaArmann \\ Fritz-Haber-Institut der Max-Planck-Gesellschaft, Faradayweg 4-6, 14195 Berlin, Germany \\ *Corresponding author: passler@fhi-berlin.mpg.de
}

Received 18 September 2019; posted 18 September 2019 (Doc. ID 377766); published 1 November 2019

\begin{abstract}
In our paper J. Opt. Soc. Am. B 34, 2128 (2017) in Section 2.C, the calculation of the layer-dependent electric field distribution is only valid for media with permittivity tensors that are diagonal in the lab frame, i.e., nonbirefringent media. This erratum corrects Section 2.C such that the electric field distribution in birefringent media is calculated correctly. Further, Eqs. (20) and (33)-(36) are corrected. The associated MATLAB implementation has been updated. (0 2019 Optical Society of America
\end{abstract}

https://doi.org/10.1364/JOSAB.36.003246

\section{A.3. Transfer Matrix with Treatment of Singularities}

The electric field vectors $\vec{\gamma}_{i j}$ of the four eigenmodes $j$ in each layer $i$ have been reported with an error in the components $\gamma_{i 13}$ and $\gamma_{i 33}$. The correct components are [1]

$$
\begin{aligned}
\gamma_{i 13} & = \begin{cases}-\frac{\mu_{i} \varepsilon_{i 31}+\xi q_{i 1}}{\mu_{i} \varepsilon_{i 33}-\xi^{2}}, & q_{i 1}=q_{i 2} \\
-\frac{\mu_{i} \varepsilon_{i 31}+\xi q_{i 1}}{\mu_{i} \varepsilon_{i 3}-\xi^{2}}-\frac{\mu_{i} \varepsilon_{i 32}}{\mu_{i} \varepsilon_{i 33}-\xi^{2}} \gamma_{i 12}, & q_{i 1} \neq q_{i 2}\end{cases} \\
\gamma_{i 33} & = \begin{cases}\frac{\mu_{i} \varepsilon_{i 31}+\xi q_{i 3}}{\mu_{i} \varepsilon_{i 33}-\xi^{2}}, & q_{i 3}=q_{i 4} \\
\frac{\mu_{i} \varepsilon_{i 31}+\xi q_{i 3}}{\mu_{i} \varepsilon_{i 33}-\xi^{2}}+\frac{\mu_{i} \varepsilon_{i 32}}{\mu_{i} \varepsilon_{i 33}-\xi^{2}} \gamma_{i 32}, & q_{i 3} \neq q_{i 4} .\end{cases}
\end{aligned}
$$

Furthermore, in order to ensure the correct calculation of the cross-polarization reflection and transmission coefficients for birefringent media, the eigenmode field vectors $\vec{\gamma}_{i j}$ have to be normalized:

$$
\overrightarrow{\hat{\gamma}}_{i j}=\frac{\vec{\gamma}_{i j}}{\left|\vec{\gamma}_{i j}\right|}
$$

and $\overrightarrow{\hat{\gamma}}_{i j}$ replaces $\vec{\gamma}_{i j}$ in the further formalism, specifically in Eq. (22) in Ref. [2].

\section{B. Reflectance and Transmittance}

In our original work [2], we had defined the transmission coefficients $t_{s s}, t_{p s}$, and $t_{s p}$ with opposite sign compared to the original work by Yeh [3] for convenience of the previous electric field calculations. While this has no impact on the intensity enhancement $T_{k l}=\left|t_{k l}\right|^{2}$ (with $k, l=s, p$ ), the sign does matter for the corrected calculation of the electric fields (see following section). We therefore now employ the transmission coefficients following the sign convention of Yeh [3]:

$$
\begin{aligned}
& t_{p p}=\frac{\Gamma_{33}^{*}}{\Gamma_{11}^{*} \Gamma_{33}^{*}-\Gamma_{13}^{*} \Gamma_{31}^{*}} \\
& t_{s s}=\frac{\Gamma_{11}^{*}}{\Gamma_{11}^{*} \Gamma_{33}^{*}-\Gamma_{13}^{*} \Gamma_{31}^{*}} \\
& t_{p s}=\frac{-\Gamma_{31}^{*}}{\Gamma_{11}^{*} \Gamma_{33}^{*}-\Gamma_{13}^{*} \Gamma_{31}^{*}} \\
& t_{s p}=\frac{-\Gamma_{13}^{*}}{\Gamma_{11}^{*} \Gamma_{33}^{*}-\Gamma_{13}^{*} \Gamma_{31}^{*}} .
\end{aligned}
$$

\section{C. Electric Field Distribution}

In our paper [2], the four eigenmodes $j=1-4$ in each layer $i$ were assigned to be $p$-polarized $(j=1,3)$ and $s$-polarized $(j=2,4)$ modes (transmitted and reflected, respectively, where the plane of incidence is in the $x-z$-plane). This is true for any dielectric tensor that is diagonal in the lab frame (in the following referred to as non-birefringent), where for the $p$-polarized mode $E_{y}=0$ and for the $s$-polarized mode $E_{x}=0$. For birefringence, however, the eigenmodes are no longer 
$p$ - and $s$-polarized, but can be distinguished as ordinary $(o)$ and extraordinary $(e)$ modes, both featuring $E_{x} \neq 0$ and $E_{y} \neq 0$. Therefore, in the case of a birefringent substrate, the transmission coefficients [Eqs. (33)-(36) in [2]] must be labeled $t_{p o}, t_{s e}, t_{p e}, t_{s o}$ instead of $t_{p p}, t_{s s}, t_{p s}, t_{s p}$, and they describe the transmission into the ordinary/extraordinary mode for $p$ - or $s$-polarized incident light, respectively. As a consequence, the electric field components $E_{x}, E_{y}$, and $E_{z}$ cannot be calculated anymore directly from the transmission coefficients as shown in Eqs. (39)-(41) in [2]. In the following, we present a generic solution for the calculation of the electric field vectors that is applicable to non-birefringent as well as arbitrarily birefringent media.

Using the interface and propagation matrices $\mathbf{L}_{i}$ and $\mathbf{P}_{i}$, respectively, the four-component electric field vector as defined in Eq. (23) in [2] can be projected to any point in the multilayer system. The four components describe the amplitudes of the $p$ and $s$-polarized modes in non-birefringent, and the amplitudes of the ordinary and extraordinary modes in birefringent media. Therefore, in the following we will refer to the four-component vector as the amplitude vector. Utilizing the corrected transmission coefficients given by Eqs. (33)-(36*), the amplitudes of the modes, i.e., the amplitude vector $\vec{E}_{N+1}^{+}$, in the substrate at the interface with layer $N$ [see Fig. 1(a) in [2]] can be calculated separately for $p$ - and for $s$-polarized incident light as follows:

$$
\begin{aligned}
& \left(\vec{E}_{N+1}^{+}\right)_{p \text { in }}=\left(\begin{array}{c}
E_{\text {trans }}^{p / o} \\
E_{\text {trans }}^{s / e} \\
E_{\text {ref }}^{p / o} \\
E_{\text {refl }}^{s / e}
\end{array}\right)=\left(\begin{array}{c}
t_{p(p / o)} \\
t_{p(s / e)} \\
0 \\
0
\end{array}\right) \\
& \left(\vec{E}_{N+1}^{+}\right)_{s \text { in }}=\left(\begin{array}{c}
E_{\text {trans }}^{p / o} \\
E_{\text {trans }}^{s / e} \\
E_{\text {refl }}^{p / o} \\
E_{\text {refl }}^{s / e}
\end{array}\right)=\left(\begin{array}{c}
t_{s(p / o)} \\
t_{s(s / e)} \\
0 \\
0
\end{array}\right),
\end{aligned}
$$

where the reflected components are set to zero, since no light source is assumed to be on the substrate side of the multilayer system. Further note that we here conveniently go back to the field sorting according to Eq. (23) in [2], allowing one to directly reuse any interface and propagation matrices that were calculated along the way to the full transfer matrix $\boldsymbol{\Gamma}_{N}$. As it is shown in Fig. 1(a) in [2], the amplitude vectors on both sides of an interface are connected by the interface matrix $\vec{E}_{i-1}^{-}=\mathbf{L}_{i} \vec{E}_{i}^{+}$. Furthermore, the propagation through layer $i$ is given by the propagation matrix $\mathbf{P}_{i}$, which can be evaluated $z$-dependently:

$$
\begin{aligned}
& \vec{E}_{i}(z)=\mathbf{P}_{i}(z) \vec{E}_{i}^{-} \\
& \quad=\left(\begin{array}{cccc}
e^{-i \frac{\omega}{c} q_{i 1} z} & 0 & 0 & 0 \\
0 & e^{-i \frac{\omega}{c} q_{i 2} z} & 0 & 0 \\
0 & 0 & e^{-i \frac{\omega}{c} q_{i 3} z} & 0 \\
0 & 0 & 0 & e^{-i \frac{\omega}{c} q_{i 4} z}
\end{array}\right) \vec{E}_{i}^{-},
\end{aligned}
$$

with $0<z<d_{i}$ being the relative $z$-position in layer $i$. As it is indicated by the black arrows in Fig. 1 in [2], starting from $\vec{E}_{N+1}^{+}$, interface matrices $\mathbf{L}_{i}$ and propagation matrices $\mathbf{P}_{i}$ are used to subsequently propagate the wave towards the incident medium. In the reverse direction, the inverse propagation matrix $\mathbf{P}_{N+1}{ }^{-1}$ allows one to calculate the $\vec{E}$-fields in the substrate. By this means, the four mode amplitudes $E_{\text {trans }}^{p / o}, E_{\text {trans }}^{s / e}$, $E_{\mathrm{refl}}^{p / o}$, and $E_{\mathrm{refl}}^{s / e}$ are obtained as a function of $z$ within each layer. In contrast to the originally published paper, these calculations are performed seperately for $p$ - and $s$-polarized incident light, ensuring that birefringence is treated correctly.

In order to obtain the three-component $(x, y, z)$ electric field vectors for each of the four modes $j$, the four mode amplitudes are multiplied with their respective normalized eigenmode vector $\overrightarrow{\hat{\gamma}}_{i j}$ [Eq. (20) in [2] and Eq. (E1)]. This yields the full electric fields of the four modes for $p$ - and $s$-polarized incident light seperately, replacing Eqs. (39)-(41) in [2]:

$$
\begin{aligned}
& \left(\vec{E}_{\text {trans }}^{p / o}\right)_{p / s \text { in }}=\left(E_{\text {trans }}^{p / o}\right)_{p / s \text { in }}\left(\begin{array}{l}
\hat{\gamma}_{i 11} \\
\hat{\gamma}_{i 12} \\
\hat{\gamma}_{i 13}
\end{array}\right) \\
& \left(\vec{E}_{\text {trans }}^{s / e}\right)_{p / s \text { in }}=\left(E_{\text {trans }}^{s / e}\right)_{p / s \text { in }}\left(\begin{array}{l}
\hat{\gamma}_{i 21} \\
\hat{\gamma}_{i 22} \\
\hat{\gamma}_{i 23}
\end{array}\right) \\
& \left(\vec{E}_{\text {refl }}^{p / o}\right)_{p / \text { in }}=\left(E_{\text {refl }}^{p / o}\right)_{p / s \text { in }}\left(\begin{array}{l}
\hat{\gamma}_{i 31} \\
\hat{\gamma}_{i 32} \\
\hat{\gamma}_{i 33}
\end{array}\right) \\
& \left(\vec{E}_{\text {refl }}^{s / e}\right)_{p / s \text { in }}=\left(E_{\text {refl }}^{s / e}\right)_{p / s i n}\left(\begin{array}{l}
\hat{\gamma}_{i 41} \\
\hat{\gamma}_{i 42} \\
\hat{\gamma}_{i 43}
\end{array}\right),
\end{aligned}
$$

for each layer $i$ and as a function of $z$, where we have omitted the index $i$ and the $z$ dependence for the sake of readability.

Within each layer, there are only two possible shapes of the amplitudes of these eigenmodes: they either describe a (damped) sinusoidal propagating wave, or an exponentially decaying evanescent wave. In order to get the full electric field vectors present in the multilayer system, the electric field components of all four modes, the forward (transmitted) and backward (reflected), $p / o$ and $s / e$, respectively, have to be summed up. Note that by utilizing the eigenmode vectors $\overrightarrow{\hat{\gamma}}_{i j}$, it is no longer necessary to consider phase flips upon reflection. Furthermore, all three components of the electric field vectors are calculated correctly even for birefringent media, and the fields are obtained seperately for $p$ - and $s$-polarized incident light. The associated MATLAB code [4] has been updated, implementing the corrected formulas. Additionally, Jeannin implemented the formalism in Python [5].

Furthermore, in contrast to the reflection coefficients $r$ which allow one to directly calculate the reflectance $R=|r|^{2}$, the transmittance $\mathcal{T}$ cannot be calculated directly from the transmission coefficients, i.e., in general, $\mathcal{T} \neq|t|^{2}$ (only when the last material is vacuum the transmittance is $\mathcal{T}=|t|^{2}$ ). The correct calculation of the transmittance and absorption in a multilayer system of arbitrarily anisotropic media-including birefringence-will be treated elsewhere [6].

Acknowledgment. The solution for the calculation of the electric field distribution in birefringent media presented in this erratum has been triggered by the feedback and discussion with various collegues in the community. For their contribution we 
therefore thank, in chronological order, Kevin Vynck (Institut d'Optique d'Aquitaine, Bordeaux), Ota Kunt (Technische Universität Dresden), Quianxiang Ai (University of Kentucky), Lloyd Bumm (University of Oklahoma), Mathieu Jeannin (Ecole Normale Supérieure de Paris), Yujin Tong (Fritz Haber Institute, Berlin), and Kramer Campen (Fritz Haber Institute, Berlin).

\section{REFERENCES}

1. W. Xu, L. T. Wood, and T. D. Golding, "Optical degeneracies in anisotropic layered media: Treatment of singularities in a $4 \times 4$ matrix formalism," Phys. Rev. B 61, 1740-1743 (2000).
2. N. C. Passler and A. Paarmann, "Generalized $4 \times 4$ matrix formalism for light propagation in anisotropic stratified media: study of surface phonon polaritons in polar dielectric heterostructures," J. Opt. Soc. Am. B 34, 2128-2139 (2017).

3. P. Yeh, "Electromagnetic propagation in birefringent layered media," J. Opt. Soc. Am. B 69, 742-756 (1979).

4. N. C. Passler and A. Paarmann, "Generalized $4 \times 4$ matrix algorithm for light propagation in anisotropic stratified media (Matlab files)," 2019, https://doi.org/10.5281/zenodo.601496.

5. M. Jeannin, "Generalized $4 \times 4$ matrix algorithm for light propagation in anisotropic stratified media (Python files)," 2019, https://doi.org/10.5281/zenodo.3417751.

6. N. C. Passler and A. Paarmann, "Layer-resolved transmittance and absorption of light propagating in arbitrarily anisotropic stratified media" (to be published). 